\title{
Variants of DSR Protocol and their Contrasting Features
}

\author{
Priyanka Singh \\ P.S.I.T. \\ Kanpur, U.P. (208020) \\ A.K.T.U. Lucknow.
}

\author{
Palak Baaga \\ P.S.I.T \\ Kanpur, U.P.(208020) \\ A.K.T.U. Lucknow
}

\author{
Saurabh Gupta \\ P.S.I.T. \\ Kanpur, U.P.(208020) \\ A.K.T.U. Lucknow
}

\begin{abstract}
Energy efficient routing protocols have been the major topic of research these days. The main reason behind the wider research on the same is the limitation of mobile adhoc networks. The mobile adhoc network consist of nodes which have unlimited mobility level within the range, and most importantly limited battery capacity. These drawbacks of nodes harm the nodes itself and even the network's capacity they belong to. So as a result of this many routing protocols have been optimized and converted into energy efficient routing protocols. Out of the present protocols DSR is considered as the best protocol when conservation of energy is the need. This paper describes why DSR is considered as the best energy efficient algorithm. It also discusses the original DSR in brief and elaborating the strategies of optimization. This paper describes many variants of energy efficient DSR protocol. Each of the protocol uses different mechanism of optimization, but having the same global goal of effective energy utilization.
\end{abstract}

\section{Keywords}

MANETs, DSR, EEDSR, EDDSR, ESDSR, MEDSR, GEAR, LEAR, 0-DSR, I-DSR, PADSR, E-DSR.

\section{INTRODUCTION}

A mobile adhoc network is infrastructure less network with the nodes which are mobile and each node is associated with the battery of limited power and capacity. So the major issue of the network capacity was the limited energy of the nodes involved in the mobile adhoc network. Although the energy is provided in a limited amount but the use of energy in MANETs is almost every second. The energy in the network is utilized in the case of transmitting a packet, on receiving a packet irrespective of the fact that packet can be discarded it is still utilized. Hence the energy utilization takes place both in the sleep and active mode. There have been protocols like DSR, AODV, TORA, ZRP and DSDV which functions to perform routing in a mobile adhoc network. Out of the above mentioned protocols some are reactive, proactive and some hybrid. Keeping the limitations of MANETs in mind these protocols have been optimized and converted into energy aware routing protocol. These protocols use energy efficiency as the major metric along with minimum hop as used earlier. Some of these protocols are power saving medium access control layer protocols or energy efficient routing algorithms or power sensitive network architectures. On the study basis, DSR PROTOCOL has shown the best optimization result. The results in terms of average energy consumption, average end to end delay, average drop packets is the least[4]. So it is very clear that the DSR protocol is the most favorable protocol in term of finding power aware routes as it is considered as a protocol that maintains itself [2]. This protocol is adapted as the easiest protocol in finding the best possible route both in terms of energy consumption as well as in terms of distance and bandwidth. This protocol works in two steps. First is the route discovery and the second is the route maintenance. In the process of route discovery the route request RREQ packets are flooded from source to all the nodes. All the intermediate nodes that have the path from the source to the destination send the route reply message to the source. These multiple paths from the source to the destination is stored in the route cache. Then the process of route maintenance occurs only when the packet is actually sent from the source to the destination. The basic focus of the route maintenance is to ensure that the optimum route which was found in the route discovery process remains optimum even if the topology of the network changes [2]. This deals with the detection of change in the network topology. If the path from the source to the destination is broken then a route error packet RERR is send from the intermediate nodes in order to clear and update the route cache of all the other involved nodes.

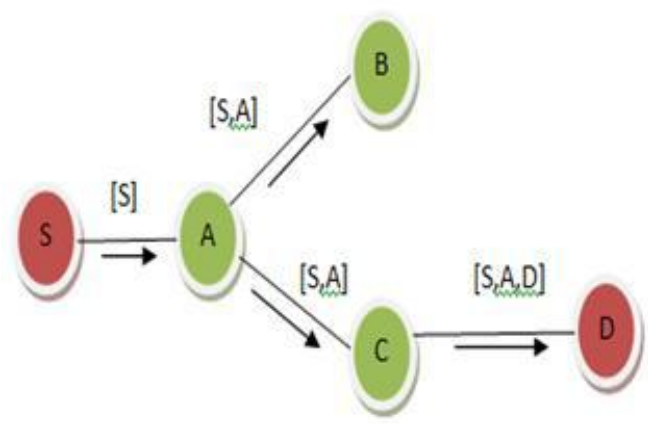

Fig 1: Route Request

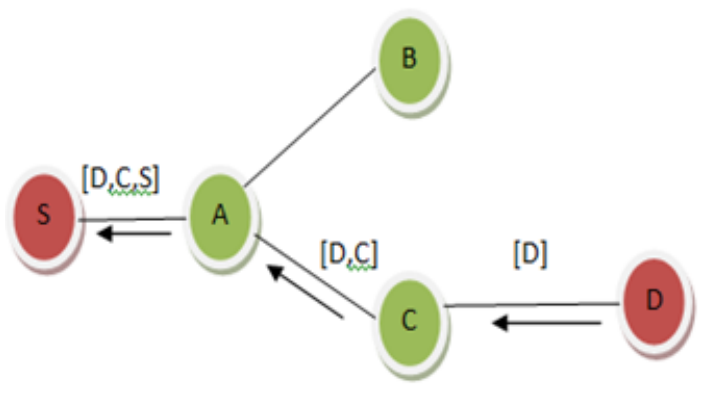

Fig 2 : Route reply[7]

The above described DSR protocol is limited in terms of consumption of power as it requires flooding of route requests packets regularly. This consumption of power makes the network limited in terms of power and energy. Hence there is need to optimize the existing protocol to make the DSR protocol as one of the best ENERGY EFFICIENT ROUTING PROTOCOL[1]. The most important feature of the energy efficient protocol is to manage the energy, its usage in order to increase the lifetime of the nodes and eventually of the network. 


\section{WHY DSR??}

A basic and genuine question that arises in the mind is that why only DSR is considered as the best protocol to optimize when it comes to energy efficiency. There are many protocols that have been converted into their energy efficient version but still DSR is preferred when energy efficiency is the issue There are many reasons to the above stated fact-

- DSR provides route caching facility. The route caches are used to save the path that has ever been discovered by the node. Hence before the initiation of route discovery process each node checks its cache for the availability of the route. The presence of route cache reduces overheads.

- $\quad$ DSR protocol on optimization has shown better average delay than other protocols on optimization.

- The strength of DSR protocol lies behind the fact that route discovery process occurs only when the path or the route is needed.

- $\quad$ DSR does not support the use of beacons.

- Because of the presence of route cache the DSR protocol provides multiple routes to the destination which are all saved in route cache of respective nodes.

- $\quad$ DSR has proven better average drop packets.

- DSR protocol does not even requires symmetric links.

\section{METHOD OF OPTIMIZATION}

There are several methods of optimization of DSR protocol to convert it into a Energy efficient routing protocol-:

- The flooding of the route request packets can be done periodically. A threshold value of energy is set after every time $\mathrm{T}$ and is considered for flooding the packet. The threshold value of the nodes is used to decide either to flood the packet or not.

- The other mechanism can be the one in which the nodes are expected to show gratitude towards the source node by sending the shortest path from the source to destination if available to them and unavailable in the route cache of the source node and therefore, unknown to the source node.

- The other method is avoiding "route reply storms" i.e the route reply packet coming from the nearer nodes are given more importance and high priority as compared to the far away nodes.

- "Packet salvaging" is another method of optimization in which the packet is not discarded by the intermediate node even if the rout is broken if another path to the destination is present in the route cache of that particular node.

\section{VARIANTS OF DSR PROTOCOL.}

From the above paragraphs it has been made clear that DSR is one of the best protocol to conserve energy when optimized. The following paragraph deals with the description of variants of DSR protocol and the mechanism of optimization used by them.

\subsection{Energy efficient DSR(EEDSR)}

The energy efficient DSR protocol[1] deals with optimization in both route discovery and route maintenance of the nodes. This protocol avoids the constant flooding of the packets by having a rigid route caching technique. The flooding of request packets is periodically done by setting a regular time interval. Only the nodes that have optimal energy for transferring the packets from itself to destination are considered in the path. The resource allotment to the nodes while transferring the packet is fixed in order to have a constant transmit power.

Minimum number of hops and remaining energy value forms the basis of the selection of the best route. Further the protocol optimizes the method of discovery and the maintenance of the route. The information about the energy is send along with the request packets which has the source address, destination address, sequence number to avoid delays. The initialization of the process takes place with same energy level of the nodes and keeping in mind minimum hop count as the metric. Moving along further various metrics such as transmit power, remaining energy level and minimal energy consumption is also taken into consideration. The algorithm works with the initial discovery process including following steps-

- If this discovery is not the first one check for the presence of the route between source and destination.

- The route cache of the node saves the entries which include the ID's of both source and destination, the energy level of the source and the threshold energy.

- On receiving the packet the intermediate node adds its information that includes its ID and the energy level and the packet is forwarded along the path available.

- Amongst the path available it chooses the best possible shortest route.

The route maintenance works under two conditions. The first is the case when the node has to be discarded because of the decrease in its energy level which goes down below thw threshold value. So this information has to be distributes to all the nodes participating in the path. Secondly, the case when the nodes crosses the maximum assigned mobility level. This constant defined mobility level decreases the emergency of route maintenance. So the route maintenance is used to deactivate those links whose nodes either crosses the mobility level or has energy level less than the threshold energy[1].

\subsection{Power aware dynamic source routing protocol(PADSR)}

Another variant of a DSR protocol is a power aware dynamic source routing protocol which is based on the transmission control approach. In this approach the protocol functions to minimize the transmission power involved in transferring the packets from the source to destination of all the nodes involved in the transmission. This is a step by step protocol which functions in minimizing the power consumption at each node and the total power consumption automatically. The life of the network is improved by involving the nodes which are rich in power and discarding those with less power as they may utilize more amount of energy. Dynamic power control approach is applied instead of fixed power control approach by calculating the power cost and other parameters at each link and node. This protocol works by allowing several transmissions to occur simultaneously which increases the capacity of the network. An additional field called as minpower field is appended along with the existing fields. This field is used to store the remaining power of the battery. By using this field the connections amongst the link which breaks before the expected time is avoided. The value of this field is 
updated on receiving the request packet by comparing the existing value of the field by the remaining energy of the nodes till the packet reaches the destination. In case of multiple routes the route with maximum minimum power field is selected such the nodes which can expire soon are neglected.

The calculation of the power at each node is done by th formula-

$P(n)=p(t)-p(r)+p(t h)+p(m)$

Where,

$\mathrm{P}(\mathrm{n})=$ new transmission power

$\mathrm{P}(\mathrm{t})=$ power during transmission of the request packet

$\mathrm{P}(\mathrm{r})=$ power during receiving of the request packet

$\mathrm{P}(\mathrm{th})=$ threshold value of the power

$\mathrm{P}(\mathrm{m})=$ the power utilized to overcome the unreliable links[3].

The calculated power is stored and as this power is taken as the minimum power to transmit and receive packets throughout the network successfully. The intermediate nodes rebroadcasts the packet with the maximum power and this chain goes till the destination reaches the destination. The destination may receive more than one request packet so it selects the route with the maximum power so that the communication between the source and destination goes on for a longer period of time. The rest of the request packets are discarded to avoid overheads. This process continues between each and every source destinations pair to get a successful communication. So by this protocol the energy is conserved both at the node level as well as the network level [2].

\subsection{Enhanced-DSR(E-DSR)}

Another version of an energy aware DSR protocol is EDSR[4]. Rather than focusing on minimum utilization of energy during transmission and receiving as in the other versions, this version focuses on using the route with maximum energy in order to transfer the packet. This protocol introduces a slight change in the route discovery process. In the step of discovery of a energy efficient route only the nodes that are the neighbor nodes as well as energy efficient participate in the process. As an energy efficient neighboring node receives a request packet with the source id, destination id, and sequence no and total energy consumption field, it checks its cache for the availability of the path. If the path to the destination is unavailable the packet is rebroadcasted by the same fields within the packet by the intermediate node just by updating the total energy field with its local energy field in it. This process continues until the packet either reaches to the destination node or the intermediate energy efficient node with the complete information to the destination node. As in the original DSR each of the nodes contains a cache which minimizes the use of energy if the path to destination is available. The path is sent in a route reply packet to the source. And in the case of unavailability the packet is forwarded. One of the packets that reach the destination first is the packet with the complete route to the source. The source node on receiving the reply caches the path to destination in the local cache and uses the route to communicate with destination. The process of route maintenance is used as the process to ensure the validity of the route discovered in the route discovery process. The message such as acknowledgement validates the route found. This acknowledgement packet is send by the energy efficient neighboring node to the node from which they have received the request packet. This packet is sent after the neighboring node has forwarded the packet to the next hop. The use of the route error packet is same as that in the original DSR that is, the route error message shows the change in topology, breaking of the link, and requests the source to start the new route discovery process. The route error packet also gives signal to the nodes to update their caches by removing the concerned routes. This way the protocol focuses on the more energy nodes in the discovery process and helps the network against the drop of energy due to limited battery.

\subsection{Improved DSR(IDSR)}

This version of DSR[5] works independent to the neighboring nodes. This protocol does not use the information about the neighboring nodes such as their route cache or their local energy level. The intermediate nodes on receiving the request packets from the source, checks the energy level of the packet. After the calculation of the energy level "back off time" is calculated. This back off time depends on the receiving power of the RREQ packet inversely. If the receiving power of the request packet is more, then the back off time is expected more, and if the receiving power of the packet is less then, the back off time is more. After the above calculation all the intermediate nodes helps in transfer of the packets from the source to destination. The main attraction of this protocol is "time to live" condition. This condition manages the flow of the algorithm. After the calculation of TTL the value of TTL is checked. If the value is less than 0 the packet is dropped. If the value of TTL is greater than 0 the id is compared to check if the receiving node is destination or an intermediate node. If the node is itself a destination it does three things. First, it calculates the total delay. Second, energy level of the node on that particular path is detected. Third, a RREP packet is send to source.

In the case of intermediate node receiving a packet the values inside a request packet is updated and the RREQ packet is further broadcasted[5].

\subsection{Energy secure DSR protocol(ESDSR)}

The main contrast of ESDSR[7] protocol to others is rather than focusing on the minimum hop count metric it focuses on the method of finding the way that includes the nodes which have greater residual energy than the set threshold value. So, calculating the residual energy at every node forms the basis of this protocol. This ensures that at any instant of time every intermediate node in the path has energy more than the threshold energy. As a result of this the discovered route in the route discovery process consist of high energy route, and hence the sum of the residual energy of the associated route is high. This ensures that the association of communication will go long without any interrupting failure due to decrease in energy. So the process of route discovery lets the RREQ packet reach the destination with maximum amount of left over energy. This protocol is optimized by adding some features which were neglected in the original DSR protocol. The loss of packets in the original DSR was thought because of congestion or out of mobility range. But the focus of ESDSR is on energy. The loss of packet in this protocol is thought only because of loss of energy or less residual energy, neglecting all the reasons such as of congestion or mobility. As the process continues the energy of the nodes is used up continuous. Each node has the responsibility of tracing its own energy. As any node discovers that its energy is less than or equal to the threshold energy it reminds and informs all the other participant nodes that it is no more eligible to forward the packets. This step is carried out with the help of an "alert" message. The corresponding node also informs the source 
node that the source node needs to find another node to destination. The threshold value of energy for this protocol is taken as $1 \mathrm{~J}$. so as any node's energy goes below $1 \mathrm{~J}$ it sets the value " 1 " to low energy field in the error control message. So as the neighboring nodes receive the error control message they simply discard the node and thus the route. The source then finds out the new route to the destination by the same route discovery process. The attracting feature of this protocol is that it does not let any node die out completely because of the loss of energy. The ESDSR protocol both saves and endures the energy[7].

\subsection{Energy dependent DSR protocol(EDDSR)}

The working of EDDSR[6] protocol depends entirely upon the nodes of the network. It is completely the wish of anode that it wants to participate in the network or not. The intermediate nodes use the value of their current energy to actually find their willingness to participate in the route. The route discovery process takes place with slight modification. Each node ' $n$ ' performs calculation to find its own RBP (residual battery power). If the value of RBP is greater than the specified value, allows the node to participate and performs all the functions of a DSR node. If the value is not good enough the nodes delays the forward rebroadcasting of the message. The amount of delay is calculated by PLT (predicted life time). The predictive life time is inversely proportional to the delay. The value of PLT is a ratio of RBP and DR (drain rate) of the particular node.

\section{$\mathrm{PLT}=\mathrm{RBP} / \mathrm{DR}$}

The drain rate of a node is the energy a particular node consumes in every second. The amount of time a node will take to complete get exhaust is given by predicted life time. So the nodes whose predicted life time is less do not participate in the route discovery process. The RREQ packet coming from the nodes with less predicted lifetime is discarded by the receiving nodes which are nearer to the destination node, because of the fact that only one packet needs to be reached to the destination. The process of route maintenance is also a little different and modified. All the nodes whose energy falls below the threshold energy informs about their current energy level by using RERR packet. This specifies the source node to start the route discovery find another route to the destination.

\subsection{Optimized DSR (O-DSR)}

The o-DSR[9] protocol aims at solving two important issues of MANETs simultaneously. First, is the congestion if it exists in the network and second, is to increase the life of the nodes and thus the network capacity. This protocol is basically optimized by optimizing the route selection process. The route discovery process in the basic DSR protocol requires a storm of broadcasting of RREQ messages. If there are a total of $n$ nodes each having an average of $m$ neighbors then the total messages in the network broadcasted will be $\mathrm{n} * \mathrm{~m}$. This overhead due to excessive number of broadcasted message is reduced by o-DSR. The optimization is done by dividing the step of route discovery into two phases. The first phase is to select the list. The list contains the nodes of the zone that are one step to destination. The parameters such as network size, neighboring nodes are used to find the node list. The second phase is to update the list. This phase is an important phase of the protocol as the mobility of the mobile nodes is the biggest issue. So update of the list takes place by the help of an "alert" message. This alert message is send by the node whose link is broken to the neighbouring nodes to give them a message to delete the lists that contain the left node. Any node that receives the alert message deletes the list that contains that node and passes the same message to its neighbors[9].

\subsection{Minimum energy dynamic source routing protocol(MEDSR)}

MEDSR[10] protocol; a variant of DSR protocol focuses on the way to minimize the use of various control messages as used by other variants. It manages to save energy by using the control messages of the original DSR protocol. The steps of MEDSR are a little different from that of original DSR. The first one is "route discovery" and the other is "link by link power adjustment". The process of route discovery is performed in two levels. The first level says that the source initiates the process of route discovery by using a power of low level. It finds the connection between itself and the destination. If it does not find the route, thee process is reattempted using the same power level. Even if the route is not found it assumes that the destination is unreachable. The second level increases the power to a little higher than the power of the first level and restarts the route discovery process. Meanwhile the route discovery process takes place by one of the above levels, the link by link power adjustment of transmit power is done. This adjustment by using a route reply packet of the network layer decreases the use of energy. The specialty of the protocol is the guaranteed discovery of route in the low or the high power and minimum use of energy as even in the high power level link by link adjustment decreases the transmit power[12].

\subsection{Global energy aware routing protocols(GEAR)}

The protocol named as GEAR[8] protocol adds up the information contained in the route request packet. Along with the request message it adds the remaining energy level, its identity in the packet and broadcasts the packet to the neighboring nodes. This added information in the request packet makes the job of the destination easier. The destination needs to select the best route. So this remaining energy information in all the received packets forms the basis of the selection and ensures the selection of best route with maximum energy. The route which is selected is not always the best route because the packet with the minimum energy may arrive after the specified time duration. The GEAR protocol has two major disadvantages. The first one being, the inefficiency of using the route cache and the second, is the blocking property. The reason of unusable route cache leads to flooding of messages. The other problem is the waiting time of the request messages. The destination waits for the best RREQ message. At times the route selected in the short period of time is not always the best route. And, waiting too long affects the response time. These two problems are solved in the next explained variant.

\subsection{Local energy aware routing protocol(LEAR)}

This variant of a DSR protocol balances the usage of energy in a real time environment and overcomes the problem of GEAR protocol. It allows those nodes to participate in the route discovery process whose remaining battery power is more than the threshold energy. This condition ensures that all the nodes in the route discovery process are highly rich in terms of battery power. So on receiving the packet the node checks its energy with the threshold energy, if the energy is more than the threshold energy it takes part in the route 
discovery process and forwards the packet, otherwise the packet is dropped. The protocol shines in one way. If at any instant the route is discarded because of one node in the route lacks in fulfilling the condition of energy more than the threshold energy the protocol reattempts in order to maintain the previously established route. For this, the protocol sends the same request packet with an increased sequence number. The corresponding node that was responsible for discarding of the packet receives the packet with increased sequence number; it manages the threshold value for the completion of the route. This variant is more efficient in terms of energy as it saves 35\% more energy than DSR. The DSR protocol allows individual nodes to use different amount of energy while LEAR[11] maintains the constant amount of energy which can be used by all the nodes [8]. The advantages of LEAR protocol are it balances the amount of energy, does not block, and integrates with the MANETs easily.

\section{CONCLUSION}

So, from the above discussions it is very clear that DSR protocol is an easiest protocol to convert it into an energy efficient routing protocol to save and secure energy of mobile nodes. There are various methods to secure energy by optimizing the protocol by any of the method. Some of the protocol gives stress on using the route with maximum amount of residual energy while others try to consume lesser amount of energy while using the route. Both described method, ultimately increase the life of the network by overcoming the limitation of MANETs. All the above described protocols are the variants of DSR protocol with the same aim of solving the issue of battery lifetime of MANETS. Hence DSR protocol is one of the best energy optimization protocol as it uses multiple parameters to enhance the efficiency of the traditional DSR protocol. Apart from the above described protocol there are many energy efficient variants of DSR protocol and there is also a scope of finding even more variants.

\section{REFERENCES}

[1] Nagende Dunican , Bulega Tonny Eddie, “An Energy Efficient Dynamic Source Routing Protocol For Mobile Adhoc Network", IJCIR.

[2] Salonee Mishra, Binod Kumar Pattanayak "Power Efficient Dynamic Source Routing “, International
Journal Of Multimedia And Ubiquitous Engineering", Volume 8, No 7, 2014.

[3] Mohamed Shalifdeen M Dhavamaniprakash , "An Energy Efficient Dynamic Source Routing For Energy Consumption In MANET" , IJIRSET , Volume 4, Special Issue 6, May 2015.

[4] Mukesh Kumar, Rahul Rishi, Dk Madan, "Comparative Analysis Of CBRP, DSR, AODV routing protocol in MANET”, IJCSE, Volume 2, Number 9, 2010.

[5] Deepti Badar, Rajendra Singh Kushwaha, "An Energy Efficient Approach To DSR Based Routing Protocol For Adhoc Network" , IJCA , Volume 118, Number 4, May 2015.

[6] JE Garcia, A Kallel, Jc Cano, R Manzona, “A Novel DSR Based Energy Efficient Routing Algorithm For Mobile Adhoc Network."

[7] Hina Khalid, Tahir Nawaz, "Energy Secure Dynamic Source Routing Protocol For MANET", IOSR-JCE, Volume 18, Issue 2, April 2016.

[8] Baisakh , “ A Review Of Energy Efficient Dynamic Source Routing Protocol For Mobile Adhoc Network", IJCA, Volume 68, Number 20, April 2013.

[9] Rjab Hajlaouvi, Savi Poul, Wissem Achour, "O-Dsr Optimized DSR Routing Protocol For Mobile Protocol For Mobile Adhoc Network", IJWMN, Volume 7, Number 4.

[10] Mohammed Tarique And Rumana Islam “ Minimum Energy Dynamic Source Routing Protocol For Mobile Adhoc Network", IJCSNS, Volume 7, Number 1, November 2007

[11] Stojmenovic I, Lin X., "Power-Aware Localized Routing in Wireless Networks", IEEE Trans. Parallel and Distributed Systems 2001; 12(11):1122-1133.

[12] Mohammed Tarique and Rumana Islam, "Minimum Energy Dynamic Source Routing Protocol for Mobile Ad Hoc Networks", IJCSNS International Journal of computer Science and Network Security, VOL 7 No. 11, November 2007. 